\title{
Additive Manufacturing as Integral Part of the Digital Solution Process - An Industrial Short Note
}

\author{
Klaus Müller-Lohmeier \\ Festo AG\&Co. KG, Esslingen, Germany \\ kum@de.festo.com
}

\begin{abstract}
Within the last years additive manufacturing established itself in the industrial environment as cutting-edge technological possibility to reduce timeto-market and manufacturing costs and to take in account even the chance of reducing resources consumption.

The paper figures that the digital development and production process chain also continues by stepping from virtual to physical world by using additive manufacturing means. This means, after all kind of sophisticated simulation based part and product optimisation, there is a time where you need parts to have a hand on, to test and verify them and even produce them in a new way to face actual economical and ecological challenges.

The additive manufacturing technology bears also a paradigm shift: from manufacturing-driven design to design-driven manufacturing. Based on a longtime practical in-house experience state-of-the-art applications with already proven practical relevance (among them is the bionic handling assistant awarded with the German Prize for Achievements in Technology and Innovation) highlight this new freedom of design.

Subsequent the current state of additive manufacturing as possibility for serial production will be discussed. There shows up both: real usable potentials and existing restrictions, too. The results of different research studies on PAbased specimen and parts manufactured by laser sintering (SLS) regarding mechanical properties, reproducibility, long-term behaviour and cost deployment will be presented. They pinpoint the huge progress of the last years but also further research needs to overcome mentioned obstacles for a continued and broader industrial application.
\end{abstract}

Keywords: Digital process chain, rapid prototyping, additive manufacturing, design-driven manufacturing, serial production, plastic laser sintering, longterm behaviour, cyclic loading.

\section{Introduction}

Festo is a leading world-wide supplier of automation technology, figure 1. As a globally-oriented and independent family company with its head office in Esslingen, Germany, it has become the performance leader in its industry over the last 50 years, thanks to its innovations and problem-solving competence in all aspects of pneumatics as well as its unique range of industrial training and education programs. Today, 
the company provides pneumatic and electric drive technology for over 300,000 customers in 200 industries world-wide both for factory and process automation as well as cutting-edge training solutions. The Festo group achieved sales revenues $€$ 2,24 billion in its fiscal year 2012 and with 16,200 employees is represented world-wide in 250 locations.

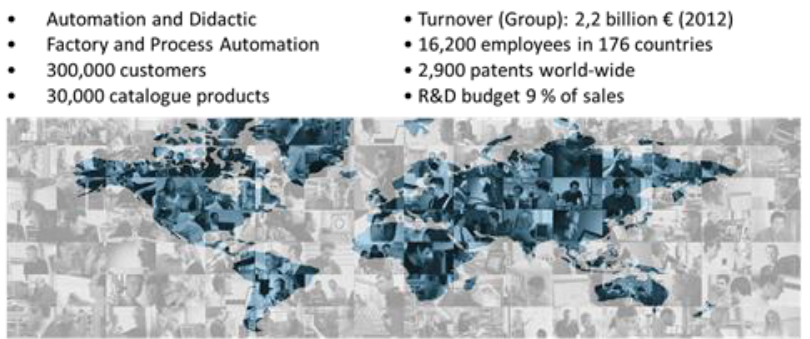

Fig. 1. Think global, act local: Festo at a glance

\section{Status of Additive Manufacturing at Festo}

Festo has a well founded practical and in-house experience in Additive Manufacturing, figure 2. Installing the first FDM machine in 1995, Festo invested continuously on this promising technology resulting in a company-wide network of currently 8 machines handling plastic materials like ABS, PC or PA12 and 2 laser melting systems for metal powders (tool and stainless steel, aluminium). The result is an annual inbound of more than 1.200 orders for additive manufactured parts (means nearly 5 orders per working day) with an admirable average door-to-door time of 4 days.

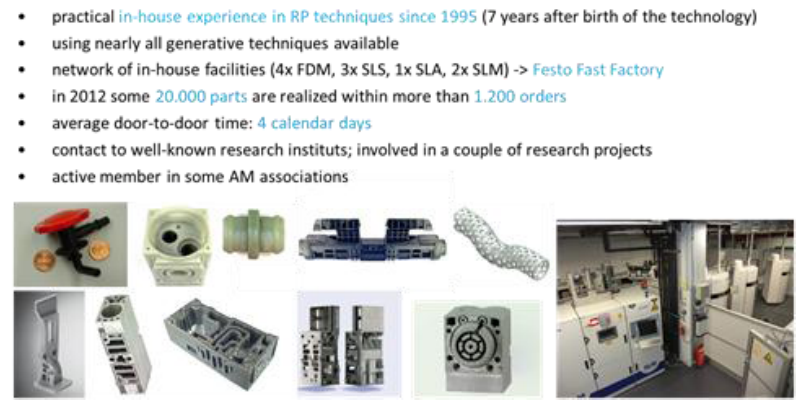

Fig. 2. Additive Manufacturing at Festo

\section{Additive Manufacturing and the Digital Solution Process}

Additive Manufacturing is a valid member of the digital solution process tools in new product development, figure 3. Starting in the virtual world ideas get first structure in 
3D CAD drawings and are optimized using various FEM software programs (mostly this might be some kind of an iterative circle). But at a certain point, there rises the wish to have some hands-on experience with the new product, to shift to the real world and feel the haptics of the parts and to carry-out functional tests.

In this case Additive Manufacturing has the advantage to use direct the CAD data and convert this in thin slices which are sended to the AM machines where a repeated deposition of material takes place building up the physical part. If design changes are created on this parts or tests show the need of further optimization subsequent reverse engineering may create a new CAD model to (re-) start the engineering process again.

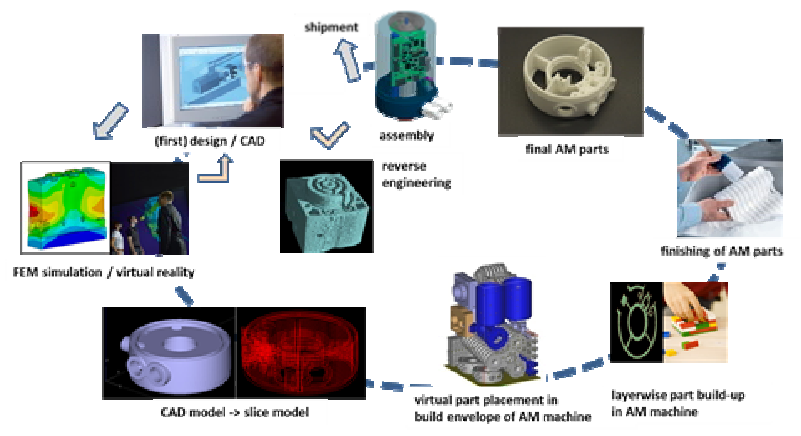

Fig. 3. Additive Manufacturing is a part of the digital solution process !

\section{Paradigm Shift in Design}

Another big benefit of applying additive techniques is the possibility to liberate design from manufacturing. The layer-wise and tool-less build-up of parts allows for example now internal structures and bending channels (to put it in other terms conventionally you will not be able to drill around 2 corners !) as well as a function integration (eg. direct co-build of hinges) and a reduction of parts and assembly efforts, figure 4 . Now manufacturing follows design leading to complete new design solutions!

A highlight of this aspect is certainly the so-called bionic handling assistant. Flexible movement and precise gripping: at first glance, the bionic handling assistant appears to be a resilient gripper arm whose structure and overall functional principle imitate an elephant's trunk. However, above and beyond its actual benefits, the bionic handling assistant is a development platform combining a wide range of technologies and components - from manufacturing concepts to series products like sensors and valves, actuators and grippers, to control technology and software for developing applications and products. This platform, as other solutions of the bionic learning network of festo, shows future concepts for tomorrow's industry and new perspectives in hazard-free human-machine cooperation. The bionic handling assistant is thus an example of how structural flexibility can be realized and adaptive soft-gripping features can be included by using additive manufacturing technology. All parts are made by plastic laser sintering leading to this unique design. 


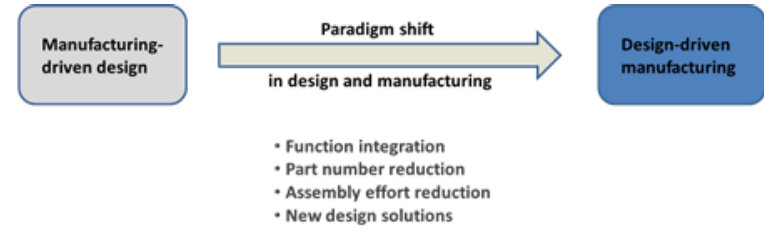

Additive Manufacturing eventually means the liberation of design from manufacturing

Fig. 4. Additive Manufacturing is more than just another manufacturing technology !
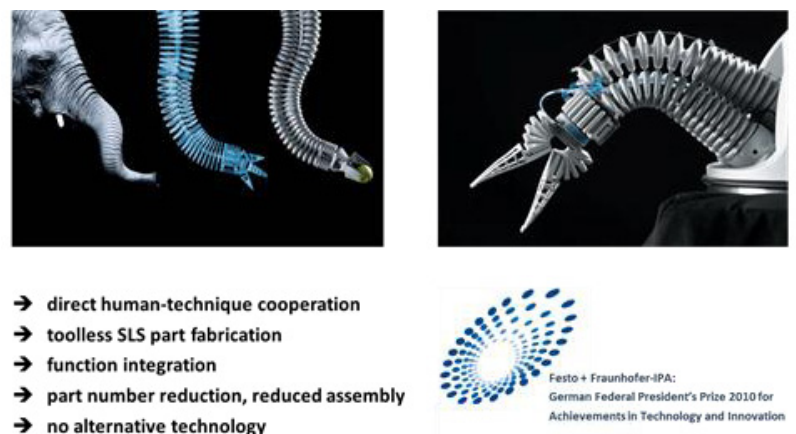

Fig. 5. Bionic Handling Assistant: Physical realisation of new handling concepts 